$11-2-2021$

\title{
Comparative Study of New and Traditional Estimators of a New Lifetime Model
}

\section{Sandeep Kumar Maurya}

Central University of South Bihar, Bihar, India, sandeepmaurya.maurya48@gmail.com

Sanjay Kumar Singh

Banaras Hindu University, Varanasi, India

Umesh Singh

Banaras Hindu University, Varanasi, India

Follow this and additional works at: https://digitalcommons.wayne.edu/jmasm

Part of the Applied Statistics Commons, Social and Behavioral Sciences Commons, and the Statistical Theory Commons

\section{Recommended Citation}

Maurya, Sandeep Kumar; Singh, Sanjay Kumar; and Singh, Umesh (2021) "Comparative Study of New and Traditional Estimators of a New Lifetime Model," Journal of Modern Applied Statistical Methods: Vol. 19 : Iss. 1 , Article 22.

DOI: $10.22237 / j m a s m / 1619482020$

Available at: https://digitalcommons.wayne.edu/jmasm/vol19/iss1/22

This Regular Article is brought to you for free and open access by the Open Access Journals at DigitalCommons@WayneState. It has been accepted for inclusion in Journal of Modern Applied Statistical Methods by an authorized editor of DigitalCommons@WayneState. 


\section{Comparative Study of New and Traditional Estimators of a New Lifetime Model}

\author{
Sandeep Kumar Maurya \\ Central University of South Bihar \\ Bihar, India
}

\author{
Sanjay Kumar Singh \\ Banaras Hindu University \\ Varanasi, India
}

\author{
Umesh Singh \\ Banaras Hindu University \\ Varanasi, India
}

In this article, we have studied the behavior of estimators of parameter of a new lifetime model, suggested by Maurya et al. (2016), obtained by using methods of moments, maximum likelihood, maximum product spacing, least squares, weighted least squares, percentile, Cramer-von-Mises, Anderson-Darling and Right-tailed Anderson-Darling. Comparison of the estimators has been done on the basis of their mean square errors, biases, absolute and maximum absolute differences between empirical and estimated distribution function and a newly proposed criterion. We have also obtained the asymptomatic confidence interval and associated coverage probability for the parameter.

Keywords: Estimation of the parameter, estimator performance criterion, Monte Carlo simulations

\section{Introduction}

To explain the real-life scenario, a number of lifetime models are available in the statistical literature for various situations. Among these, the exponential distribution is an oldest and most popular lifetime model. The reason for its popularity lies in mathematical ease in its implementation and possession of some awesome properties. But its use is restricted to those situations only where we can assume that the associated hazard rate is approximately constant. Therefore, researchers attempted to develop generalized new models having more flexibility in hazard rate and fitting. Some of the models are developed by Barlow and Proschan (1975), Kumaraswamy (1980), Mudholkar et al. (1993), Gupta and Kundu (1999), Gupta and Kundu (2001a), Gupta and Kundu (2001b), Gupta and Kundu (2007), Kumar et al. (2015), Maurya et al. (2017), etc. But the noticeable

doi: 10.22237/jmasm/1619482020 | Accepted: November 8, 2018; Published: November 11, 2021. Correspondence: Sandeep Kumar Maurya, sandeepmaurya.maurya48@gmail.com 


\section{MAURYA ET AL}

point at this stage is that nearly all of the generalizations add an extra parameter(s). No doubt, due to additional parameters model flexibility increases, but it creates difficulty in further inferences. Therefore, keeping the point of parsimony of parameter and accommodation of various hazard rates Maurya et al. (2016) proposed a new transformation method for developing new lifetime models. Needless to mention that the proposed transformation method produces a more flexible model and also preserves the baseline model properties. They considered exponential distribution as the baseline model and the new lifetime model, thus obtained, is named as Logarithmic Transformed exponential (LTE) distribution. They studied various statistical properties of the model and observed that LTE fits better than exponential, Lindley, Weibull, gamma and exponentiated exponential models for a number of data. The cumulative distribution function (cdf) and probability density function (pdf) of LTE distribution for random variable $X$ are given as:

$$
\begin{aligned}
& \mathrm{F}(x)=1-\frac{\log \left(1+e^{-\theta x}\right)}{\log 2} ; \quad \theta>0 ; x>0, \\
& \mathrm{f}(x)=\frac{\theta e^{-\theta x}}{\left(1+e^{-\theta x}\right) \log 2} ; \quad \theta>0 ; x>0,
\end{aligned}
$$

respectively. The shape of pdf of LTE distribution is more or less similar to exponential distribution for various value of the parameter. The reliability function and hazard function of LTE for specified time $t$ are given as:

$$
\begin{gathered}
\mathrm{R}(t)=\frac{\log \left(1+e^{-\theta t}\right)}{\log 2} ; \quad \theta>0 ; t>0, \\
\mathrm{~h}(t)=\frac{\theta e^{-\theta t}}{\left(1+e^{-\theta t}\right) \log \left(1+e^{-\theta t}\right)} ; \quad \theta>0 ; t>0,
\end{gathered}
$$

respectively. The mean of the LTE distribution is

$$
\mathrm{E}(X)=\frac{\pi^{2}}{\theta \log (4096)}
$$




\section{COMPARATIVE STUDY OF NEW AND TRADITIONAL ESTIMATORS}

Maurya et al. (2016) have also shown mathematically that LTE distribution has increasing hazard rate and verified this result graphically.

The median of the distribution can be obtained by putting the value $p=1 / 2$ in the $p^{\text {th }}$ quantile function $\mathrm{F}(\mathrm{Q}(p))=p$. The $p^{\text {th }}$ quantile function for the distribution is

$$
\mathrm{Q}(p)=\frac{-\log \left[2^{1-p}-1\right]}{\theta}
$$

Hence, the median of the distribution is

$$
\text { median }=\frac{\log [\sqrt{2}+1]}{\theta}
$$

It may be noted here that

$$
\frac{d}{d x} \log \mathrm{f}(x)=-\left[\frac{\theta}{1+e^{-\theta x}}\right]
$$

is a negative quantity because the quantity within bracket is always positive. So that, mode of the distribution is at the left extreme of its support i.e. at zero. Hence, we can conclude that the LTE is positively skewed distribution similar to the exponential distribution.

In the field of point estimation, various methods for estimation of the parameter are available in statistical literature, each having their own merits and demerits. To study the performance of an estimator a number of criterions have been proposed. However, researchers usually consider a method of estimation for developing the estimator of the parameter and its performance is studied on the basis of a selected criterion. It may happen that the estimator may perform better under a specific criterion but not on other criterion. Similarly, the relative performance of various estimators may differ from criterion to criterion. Therefore, in this paper, we propose to consider different estimation methods for developing the estimator for the unknown parameter and shall attempt to rank them on the basis of the study of their performances to know that which method can be said to provide better estimator overall for a small as well as large sample.

In this article, we are considering nine different estimation procedures to obtain the estimates of the parameter of LTE, which include some traditional and 


\section{MAURYA ET AL}

some new estimation methods. The estimation methods considered by us are method of moments (MME), maximum likelihood estimation (MLE) method, maximum product spacing (MPS) method, method of least squares (MLS), method of weighted least squares (WLS), method of percentiles (MOP), method of Cramervon-Mises (MCVM), method of Anderson-Darling (ADE) and right-tail AndersonDarling (ADRT) method.

For the study of the performances of the estimator, obtained by above methods, we have considered four traditional criterions namely bias, mean square error (MSE), absolute difference (Dabs) between estimated and true distribution function, maximum absolute difference (Dmax). In addition to these, we propose to use a new criterion for checking the performance of estimators which is based on the concept of difference between the estimated and true Shannon entropies. It is further proposed to estimate the asymptotic confidence interval, coverage probability, average length of confidence intervals and average estimates of the parameter of LTE.

The main objective of the present article is to investigate which method of estimation suits most for the point estimation of the parameter of the considered distribution. It further aims to check whether the performance of the estimators remains unaltered or not for the newly proposed criterion based on entropy. It has been observed that the estimators of the parameter cannot be expressed in closed form. Therefore, we have used non-linear maximization method to compute them using $\mathrm{R}$ software ( $\mathrm{R}$ Core Team, 2018). The rest of the paper is organized as follows: the different methods of estimation of the parameter are described; the criteria for the performance of the estimators are provided; Monte Carlo simulation studies for the estimators are studied; asymptotic confidence interval and average estimate of parameter with coverage probability are analyzed; conclusions of the present article are given.

\section{Estimation of the Parameter}

Let us consider that we have a random sample, say $x_{1}, x_{2}, \ldots, x_{n}$, of size $n$ from LTE model and wish to estimate the unknown parameter of the distribution. Here, we have considered nine methods of estimation which are described below one by one.

\section{Method of Moments Estimation (MME)}

This is one of the oldest methods of estimation of the unknown parameter (see

Quandt \& Ramsey, 1978). The estimate of the parameter through this method can 


\section{COMPARATIVE STUDY OF NEW AND TRADITIONAL ESTIMATORS}

be obtained by equating the theoretical moments of the proposed distribution with

the sample moments. Since LTE is one parameter distribution, so that the estimate can be obtained by using first moment i.e., mean of the distribution only. If $m$ is the sample mean, it is to be equated to theoretical mean given in equation (5), hence the estimator of the parameter $\theta$, say $\hat{\theta}$, through MME can be obtained as

$$
\hat{\theta}=\frac{\pi^{2}}{m \log (4096)}
$$

Needless to mention that it is consistent estimator and asymptotically normally distributed (for details of the moment estimators, see Casella \& Berger, 2002).

\section{Maximum Likelihood Estimation (MLE)}

This is most widely used method for estimating the unknown value of the parameter. MLE is a popular method due to its properties because if it exists, it will be most efficient estimator. Invariance property is an awesome property of MLE. In this method, we find the value of the parameter for which the likelihood function is maximum (see Casella \& Berger, 2002). The logarithm of the likelihood function of $\theta$ for the observed samples is given as

$$
\log L=n \log \left(\frac{\theta}{\log 2}\right)-\sum_{i=1}^{n}\left(\theta x_{i}+\log \left(1+e^{-\theta x_{i}}\right)\right) .
$$

The MLE of the parameter is obtained by differentiating equation (9) w.r.t. to $\theta$ and equating it to zero to get the likelihood equation as

$$
\frac{\partial \log L}{\partial \theta}=\frac{n}{\theta}-\sum_{i=1}^{n} \frac{x_{i}}{1+e^{-\theta x_{i}}}=0 .
$$

This likelihood equation of $\theta$ is an implicit function, so it cannot be solved analytically; however, iterative procedure can be used to get the solution. Here, we suggest the use of Newton-Raphson method. MLE, thus, obtained would be consistent estimator and it is also asymptotically normally distributed. 


\section{MAURYA ET AL}

\section{Method of Product Spacing (MPS)}

Cheng and Amin (1983) proposed a method of estimation as an alternative method to MLE which is increasingly becoming popular nowadays. Ranneby (1984) proposed independently the same as an approximation to the Kullback-Leibler information measure. The method is briefly described as follows:

If $x_{(1)}<x_{(2)}<\ldots<x_{(n)}$ is the ordered sample of size $n$ and $\mathrm{F}\left(x_{(i)}\right)-\mathrm{F}\left(x_{(i-1)}\right)$ for $i=1,2, \ldots, n+1$ with initial conditions $\mathrm{F}\left(x_{(0)}\right)=0$ and $\mathrm{F}\left(x_{(n+1)}\right)=1$ are called spacing.

Then, we are sampling from LTE, thus, from equation (1), the spacings are obtained as follows:

$$
\begin{gathered}
D_{1}=\mathrm{F}\left(x_{(1)}\right)=1-\frac{\log \left(1+e^{-\theta x_{(1)}}\right)}{\log 2}, \\
D_{n+1}=1-\mathrm{F}\left(x_{(n)}\right)=\frac{\log \left(1+e^{-\theta x_{(n)}}\right)}{\log 2},
\end{gathered}
$$

and the general term for $i^{\text {th }}$ spacings can be given by

$$
D_{i}=\mathrm{F}\left(x_{(i)}\right)-\mathrm{F}\left(x_{(i-1)}\right)=\frac{\log \left(\frac{1+e^{-\theta x_{(i-1)}}}{1+e^{-\theta x_{(i)}}}\right)}{\log 2}
$$

for $i=2, \ldots, n$. Note that $\Sigma_{i} D_{i}=1$. The MPS estimator is that value of the parameter which maximizes the geometric mean of the spacings, i.e.

$$
G=\left(\prod_{i=1}^{n+1} D_{i}\right)^{1 / n+1}
$$

Taking logarithm of $G$, we get

$$
\log G=S_{\theta}=\frac{1}{n+1} \sum_{i=1}^{n+1} \log D_{i}
$$


Substituting the value of $D_{i}$ from (11), (12), and (13) in the above equation, we have

$$
\begin{array}{r}
S_{\theta}=\frac{1}{n+1}\left\{\log \left[1-\frac{\log \left(1+e^{-\theta x_{(1)}}\right)}{\log 2}\right]+\sum_{i=1}^{n}\left[\frac{\log \left(\frac{1+e^{-\theta x_{(i-1)}}}{1+e^{-\theta x_{(i)}}}\right)}{\log 2}\right]\right\} \\
+\frac{1}{n+1}\left\{\frac{\log \left(1+e^{-\theta x_{(n)}}\right)}{\log 2}\right\}
\end{array}
$$

Differentiating $S_{\theta}$ with respect to parameter $\theta$, we get

$$
S_{\theta}^{\prime}=\frac{1}{n}\left[\frac{-W_{1}^{\prime}(\theta)}{1-W_{1}(\theta)}+\sum_{i=2}^{n} \frac{W_{i-1}^{\prime}(\theta)-W_{i}^{\prime}(\theta)}{W_{i-1}(\theta)-W_{i}(\theta)}+W_{n}^{\prime}(\theta)\right],
$$

where

$$
W_{i}(\theta)=\frac{\log \left(1+e^{-\theta x_{(i)}}\right)}{\log (2)}
$$

and its derivative with respect to parameter $\theta$ is

$$
W_{i}^{\prime}(\theta)=\frac{-x_{(i)} e^{-\theta x_{(i)}}}{(\log 2)\left(1+e^{-\theta x_{(i)}}\right)} .
$$

It may be noted here that by equating the derivative given in (17) to zero, the equation, thus obtained, cannot be solved analytically. Therefore, we suggest to use numerical techniques to obtain the solution. It is worthwhile to mention here that Coolen and Newby (1990) and U. Singh et al. (2014a) among many others have mentioned that MPS has many properties similar to MLE, like consistency, efficiency. Further, MPS possesses invariance property. In addition to these, it is also suggested that product spacing can be used in Bayesian analysis in place of usual likelihood function. Recently, U. Singh et al. (2014b), R. K. Singh, Yadav et 


\section{MAURYA ET AL}

al. (2016) carried out a comparative study of reliability and hazard estimates obtained by using MPS and MLE for exponential and Marshall-Olkin extended exponential distributions and found that for small sample sizes MPS provided more efficient estimator than the MLE, although, for large samples, both methods perform approximately similar. See also Basu et al. (2018) in Bayesian context.

\section{Method of Least Squares (MLS)}

This is another old and popular method of estimation (see Swain et al., 1988). We know that, if there are $n$ ordered random observations from a distribution having $\operatorname{cdf} \mathrm{F}(x)$, then

$$
\mathrm{E}\left[\mathrm{F}\left(x_{(i)}\right)\right]=\frac{i}{n+1}
$$

Thus, the least squares estimator is obtained by minimizing

$$
\operatorname{MLS}=\sum_{i=1}^{n}\left(\mathrm{~F}\left(x_{(i)}\right)-\frac{i}{n+1}\right)^{2} .
$$

Putting the cdf of LTE in equation (19), we get

$$
\mathrm{MLS}=\sum_{i=1}^{n}\left(1-\frac{\log \left(1+e^{-\theta x_{(i)}}\right)}{\log 2}-\frac{i}{n+1}\right)^{2}
$$

To minimize MLS, we differentiate it with respect to $\theta$, and equate it to zero which gives the following equation:

$$
\sum_{i=1}^{n} W_{i}^{\prime}(\theta)\left[1-W_{i}(\theta)-\frac{1}{n+1}\right]=0
$$

Again, this equation cannot be solved analytically; therefore, we use numerical techniques to obtain the solution. Gupta and Kundu (2001b) used this method of estimation for estimating the parameters of generalized exponential distribution. 


\section{COMPARATIVE STUDY OF NEW AND TRADITIONAL ESTIMATORS}

\section{Method of Weighted Least Squares (MLS)}

The estimation procedure for obtaining the estimate of the parameter through WLS is similar to the procedure of MLS with a slight change that it minimizes the weighted sum of squared deviation between true and expected cdf at observed ordered sample points, where weights are inversely proportional to the $\operatorname{var}\left[\mathrm{F}\left(x_{(i)}\right)\right]$. Thus, the weighted least square estimator is obtained by minimizing the following:

$$
\mathrm{WL}=\sum_{i=1}^{n} \frac{(n+1)^{2}(n+2)}{i(n-i+1)}\left[\mathrm{F}\left(x_{(i)}\right)-\frac{i}{n+1}\right]^{2} .
$$

Putting the cdf of LTE in equation (22), we get

$$
\mathrm{WL}=\sum_{i=1}^{n} \frac{(n+1)^{2}(n+2)}{i(n-i+1)}\left[\frac{n+1-i}{n+1}-\frac{\log \left(1+e^{-\theta x_{(i)}}\right)}{\log 2}\right]^{2} .
$$

To minimize equation (23), we differentiate WL with respect to $\theta$ and equate it to zero, which results to following equation:

$$
\sum_{i=1}^{n} \frac{(n+1)^{2}(n+2)}{i(n-i+1)} W_{i}^{\prime}(\theta)\left[1-W_{i}(\theta)-\frac{1}{n+1}\right]=0 .
$$

Equation (24) is not analytically solvable; therefore, we use numerical technique to obtain the solution. R. K. Singh, Yadav et al. (2016) have remarked that WLS performs better than MLS for Marshall-Olkin extended exponential distribution and a similar remark can be seen in Gupta and Kundu (2001b) in context of estimation of parameter of generalized exponential distribution in term of mean squared errors.

\section{Method of Percentile (MOP)}

This method of estimation was used for the first time for the estimation of the parameter of Weibull distribution by Kao (1958) (see also Kao, 1959; Balakrishnan, 2014; Gupta \& Kundu, 2001b; for more details about this method). It is easy to verify from equation (1) that $x=-1 / \theta \log \left[2^{1}-\mathrm{F}(x)-1\right]$. Thus, if $p_{i}$ are the 


\section{MAURYA ET AL}

estimates of $\mathrm{F}\left(x_{(i)}\right)$, the method of percentile (MOP) considers a value of the parameter as its estimator which minimizes

$$
\operatorname{MP}(\theta)=\sum_{i=1}^{n}\left[x_{(i)}+\frac{1}{\theta} \log \left[2^{1-p_{i}}-1\right]\right]^{2}
$$

Here, we have considered because it is an unbiased estimator of $\mathrm{F}\left(x_{(i)}\right)$ (for details about the choice of $p_{i}$, see Mann et al., 1974; Gupta \& Kundu, 2001b). Now, differentiating (25) w.r.t. the parameter $\theta$ and equating it to zero, we get the estimator $\hat{\theta}$ as

$$
\hat{\theta}=\frac{\sum_{i=1}^{n}\left[\log \left(2^{1-p_{i}}-1\right)\right]^{2}}{\sum_{i=1}^{n} x_{(i)}\left[\log \left(2^{1-p_{i}}-1\right)\right]} .
$$

For the generalized exponential distribution, Gupta and Kundu (2007) recommended it for small sample size on the basis of MSE and bias criterion.

\section{Method of Cramer-von-Mises (MCVM)}

This method is a minimum distance method based on the difference between empirical and cumulative distribution functions. MacDonald (1971) proposed this method of estimation (see Choi \& Bulgren, 1968; Boos, 1981; for more details). The method of MCVM estimator of the parameter is obtained by minimizing the following function:

$$
\mathrm{M}(\theta)=\frac{1}{12 n}+\sum_{i=1}^{n}\left[\mathrm{~F}\left(x_{(i)}\right)-\frac{2 i-1}{2 n}\right]^{2} .
$$

Hence, from equations (1) and (27), we get

$$
\mathrm{M}(\theta)=\frac{1}{12 n}+\sum_{i=1}^{n}\left[\frac{2(n-i)+1}{2 n}-\frac{\log \left(1+e^{-\theta x_{(i)}}\right)}{\log 2}\right]^{2}
$$




\section{COMPARATIVE STUDY OF NEW AND TRADITIONAL ESTIMATORS}

Now, differentiating equation (28) with respect to the parameter $\theta$ and equating to zero we get

$$
\sum_{i=1}^{n}\left[\frac{2(n-i)+1}{2 n}-\frac{\log \left(1+e^{-\theta x_{(i)}}\right)}{\log 2}\right] \frac{e^{-\theta x_{(i)}}}{\left(1+e^{-\theta x_{(i)}}\right)}=0
$$

Again, this equation cannot be solved analytically, and we propose the numerical technique.

\section{Anderson-Darling Method of Estimation (ADE)}

This method is also used minimization criteria based on Anderson-Darling statistics (Anderson, 1962). The ADE method considers the value of the parameter as its estimate which minimizes the following equation:

$$
\mathrm{AD}(\theta)=-n-\frac{1}{n} \sum_{i=1}^{n}(2 i-1)\left[\log \left(\mathrm{F}\left(x_{(i)}\right)\right)+\log \left(\overline{\mathrm{F}}\left(x_{(n+1-i)}\right)\right)\right]
$$

where $\overline{\mathrm{F}}(x)=1-\mathrm{F}(x)$ is the survival function. For LTE distribution, ADE estimator of $\theta$ can be obtained by using equation (1) in (30) and equating the derivative of it to zero. Keeping the complexities of analytical solution of this equation in mind, we propose the use of numerical approximation techniques. It may be noted here that ADE method gives more weight to tail of the distribution than the central part of the distribution. For other details and properties of ADE method, refer to Boos (1982), Louzada et al. (2016), Laio (2004), etc.

\section{Anderson-Darling Right Tail Method of Estimation (ADRT)}

This method is a variant form of ADE method which gives more weight to right tail of the distribution than other parts of it. In ADRT method for estimation, we minimize the following equation:

$$
\operatorname{ADRT}(\theta)=\frac{n}{2}-2 \sum_{i=1}^{n} \log \left(\mathrm{F}\left(x_{(i)}\right)\right)-\frac{1}{n} \sum_{i=1}^{n}(2 i-1) \log \left(\overline{\mathrm{F}}\left(x_{(n+1-i)}\right)\right)
$$

The estimator of the parameter through ADRT method is obtaining by using the equation (1) in (31) and minimizing with respect to the parameter $\theta$. Luceno (2006) 


\section{MAURYA ET AL}

used this method of estimation in comparison to MCVM and ADT. Mazucheli et al. (2017) used this method of estimation for Marshall-Olkin extended exponential distribution. Bakouch et al. (2017) used this method for the estimation of the parameters of Binomial-exponential 2 distribution.

\section{Criteria of Performance of Estimators}

In the present section, we will discuss the criteria taken for the study of performances of the estimators. Since an estimator may perform better than other for one criterion but may perform worse for another criterion. Therefore, we have considered five criteria out of which four are traditional criterion and the fifth one is a newly proposed criterion for studying the performances of the estimators. The considered criterions are discussed below one by one. An estimator is said to be good, if in repeated samples, most of the times, the estimates are close to the true value of parameter.

\section{Bias}

The bias criterion is based on the concept of error in estimating the true value of parameter. An error is defined as the difference between the estimate and the true value. That's why one may desire that the method of estimation should be such that average error is least i.e., zero. In other words, the expected value of the estimator should be equal to the true value. If $T$ is an estimator of $\theta$ then the bias is defined as

$$
B=\mathrm{E}_{\theta}(T-\theta)
$$

where $\mathrm{E}_{\theta}$ stands for expectation over the sample space. Many authors including Mazucheli et al. (2017) and Tang (2014) have used this criterion.

\section{Mean Square Error}

The measure problem with bias criterion is that while calculating the average error the opposite sign error cancels each other whereas it should be added up. This led to the concept of minimization of expected square of the error called mean square error (MSE) and is defined as

$$
M S E=\mathrm{E}_{\theta}(T-\theta)^{2},
$$




\section{COMPARATIVE STUDY OF NEW AND TRADITIONAL ESTIMATORS}

which is the sum of square of the bias and the variance of estimator. MSE can also be justified under decision-theoretic approach on the ground of the quadratic loss function and is a well-established ground to check the performance of estimators in classical as well in Bayesian scenario see, Kaushik et al. (2017), R. K. Singh, Singh, and Singh (2016), and Sharma et al. (2017).

\section{Absolute Difference}

Another way of comparison can be made on the basis of differences between the cdf specified by the estimate of the parameter $\hat{\theta}$ and cdf of distribution specified by the true parameter $\theta$. Since the difference may take positive as well as negative value, thus, expected value of sum (taken over sample values) of the absolute differences forms the basis for compression and the estimator providing the least value of sum of absolute value of the differences will be the best.

\section{Absolute Maximum Difference}

This criterion is also based on the absolute difference. In this criterion, instead of considering the sum of the differences, we consider the maximum absolute difference between the value of the cdf specified by the estimate of the parameter $\hat{\theta}$ and cdf of distribution specified by the true parameter $\theta$, for the given set of data. The expectation of maximum absolute difference is called as absolute maximum difference (Dmax) and an estimation method is called to be the best for which Dmax is minimum. Rahman and Pearson (2001) mention that the Dabs and Dmax criterion are useful in case of vector-valued parameters.

\section{New Proposed Criteria}

In the present paper, we are proposing a new criterion which is based on entropy concept:

Entropy is a measure of average amount of information contained in random variable (rv) $X$. If $\mathrm{rv} X$ has the pdf $\mathrm{f}(x)$, Shannon entropy is defined as (Shannon, 1951)

$$
\mathrm{S}(\theta)=\mathrm{E}[-\log \mathrm{f}(x)]
$$

Using equation (2) and equation (33), it is easy to verify that the entropy for the considered model is 


\section{MAURYA ET AL}

$$
S(\theta)=\frac{\pi^{2}}{12 \log 2}+\frac{\log 2}{2}-\log \left[\frac{\theta}{\log 2}\right]
$$

The form of the Shannon entropy is obtained by solving the expression given by Maurya et al. (2016). It is a measure of unpredictability of state. Jaynes (1968) gave the principle of maximum entropy for estimation of the parameter based on maximization of the Shannon entropy. Liu (2007) proposed Kullback Leibler divergence (KLD) of survival functions on the entropy. Yari and Tondpour (2017) used KLD for estimating the unknown parameter and compared the estimate with MLE and estimate obtained through maximum Shannon entropy. For more details about method of maximum Shannon entropy see, Levine and Tribus (1979), V. P. Singh and Rajagopal (1986).

Considering the importance of Shannon entropy, we wish to propose a criterion based on it. Let if $S(\theta)$ be the Shannon entropy for true value of the parameter and $S(\hat{\theta})$ be the estimated Shannon entropy obtained by putting the estimate of parameter. Now, the difference between these two may be viewed as the error in estimation of the entropy due to the use of the particular method of estimation which gives the estimate as $\hat{\theta}$. Hence, one would choose that method of estimation for which this difference is minimum. The abovementioned error in estimation of the entropy will naturally vary for sample to sample thus to get a single value, we can average it over sample space. However, in doing so, negative and positive error will cancel each other. Therefore, we propose to consider the average of magnitude of the errors which may be called an absolute entropy difference (AED). Thus, an estimation method would be best for which the value of AED is least among the class of procedures providing estimators under consideration.

\section{Simulation Study}

In the present section, we have discussed the performances of the estimators based on the simulation study. For this purpose, we have generated samples of different sizes from LTE distribution for different values of the parameter $\theta$. For the generation of samples from LTE distribution, we first generated $u$ from uniform distribution over $(0,1)$ and then used the following transformation:

$$
x=-\frac{1}{\theta} \log \left[2^{1-u}-1\right],
$$




\section{COMPARATIVE STUDY OF NEW AND TRADITIONAL ESTIMATORS}

to get the random sample values from the considered model. It may be mentioned here that the exact expression of bias, MSE, and other expressions to be used for comparison of the various estimation methods cannot be obtained because most of the estimators are not in close form. Hence, simulated values of these shall be used for comparison purpose. It may also be noted here that MSE will depend on sample size $\mathrm{n}$ and the parameter $\theta$. Hence, we have considered $n=5,15,30$, and 60 because these cover the situations of very small $(n=5)$, small $(n=15)$, large $(n=30)$, and very large $(n=60)$ samples. The variation of the parameter $\theta$ considered by us is $0.2,0.5,1$, and 2. For each value of $n$ and $\theta$, we generated $N=100,000$ random samples from LTE distribution using the equation (35). For each sample, we computed all the nine estimators, namely, MME, MLE, MPS, MLS, WLS, MOP, MCVM, ADT, and ADRT and calculated bias, MSE, Dabs, Dmax, and AED as follows:

$$
\begin{gathered}
B=\frac{1}{N} \sum_{i=1}^{N}\left(\theta_{i}-\theta\right), \\
M S E=\frac{1}{N} \sum_{i=1}^{N}\left(\theta_{i}-\theta\right)^{2}, \\
D a b s=\frac{1}{N} \sum_{i=1}^{N} \sum_{j=1}^{n}\left|\mathrm{~F}\left(x_{i j}, \theta\right)-\mathrm{F}\left(x_{i j}, \hat{\theta}\right)\right|, \\
\operatorname{Dmax}=\frac{1}{N} \sum_{i=1}^{N} \max _{j}\left|\mathrm{~F}\left(x_{i j}, \theta\right)-\mathrm{F}\left(x_{i j}, \hat{\theta}\right)\right|, \quad j=1,2, \ldots, n,
\end{gathered}
$$

and

$$
A E D=\frac{1}{N} \sum_{i=1}^{N}\left|\hat{\mathrm{S}}\left(X_{i}\right)-\mathrm{S}(X)\right|
$$

The simulation results are presented in Tables 1, 2, 3, and 4. Then, the procedures of the estimation for a fixed parameter value and sample size are ranked giving rank 1 to the best one and rank 9 (as there are 9 estimation procedures under comparison) to the worst for each criterion of comparison and are shown as the superscript of the values in these tables. The sum (taken over the criterion of comparison) of the ranks for each sample size is provided in the last row titled as $\mathrm{S}$ 


\section{MAURYA ET AL}

Rank (Sum of rank). The estimation procedures are re-ranked on the basis of the $\mathrm{S}$ Ranks and these ranks are summarized in Table 5, which shows the performance rank of all the estimation procedures for variation of $n$ and $\theta$. It would be interesting at this stage to think of ranking the performance of the estimator according to sample size. For this purpose, we summed the ranked for a procedure for all the considered value of $\theta$, although such a totaling to develop a combined index for the performance of the estimator can easily criticized but keeping in mind little variation in the ranks due to the variation in $\theta$, we propose it and the results thus obtained is given in Table 6. Similarly, we can sum the ranks for the variation in sample size also to get a single value for each procedure of estimation which is provided in the last row of Table 6 and thus the final rank of each procedure can be obtained which is shown as superscript in the last row entries of Table 6.

- Tables 1, 2, 3, and 4 show values under various criteria for different methods of estimations in the variation for sample size $n$ and the parameter $\theta$ taken as $0.2,0.5,1$, and 2 respectively.

- From these tables, we note that MSE is least for MPS for all the considered values of parameters and sample sizes but under the criterion AED, Dabs, and Dmax the values are least for MLE.

- We can also observe from these tables that as the sample size increase MSE decrease rapidly, indicating that all the considered methods are consistent (see Larson, 1974).

- It may further be seen that for increase in the sample size bias decreases for all the considered estimation methods i.e., all the considered estimation methods provide asymptotically unbiased estimates.

- Tables 1-4 show that among all the considered estimation methods only MPS and MOP have negative bias.

- From the tables we may note that for fixed sample size $n$ an increase in the value of parameter $\theta$, results increase in the values of MSE and bias both.

- It is interesting to note from Tables 1-4 that each method of estimation has same ranking under the criterion AED, Dabs, and Dmax.

- In addition to the above remark, we may further note from the comparison of the Tables 1-4 that as sample size or the value of the parameter increases the rank of AED, Dabs, and Dmax either decrease or remain the same.

- On the basis of the sum of the rankings, it is observed that for small sample size $(n=5)$, MPS perform best among all the considered estimators (see 
Table 5). However, as sample size increases, the MPS estimator could not retain its superiority over other estimators. But ADRT has sum of the ranks equal to or neat to that of MPS for $n=5$ and for $n=15$, it becomes the best. For $n=30$ or more MLE is ranked the best.

- It is interesting to note that MME is always ranked below MLE but it is always next to MLE.

- It may also be noted from Table 5, that MLS occupies the bottommost position.

- On the basis of the sum of the ranks, it may be inferred that WLS always performs better than MLS. A similar result has been stated by Gupta and Kundu (2001b) on the basis of the MSE criterion.

- Among the nine estimators considered here except four estimators, namely MLE, MPS, MME, and ADRT rest five are always ranked below these having ranks between 5-9.

- From Table 6, we may note that for small sample size MPS and ADRT performs better than others. However, for increase in the sample size the overall ranking changes. The ranking of MLE improves and reverse is the trend for MPS and ARDT although the deterioration in the ranking is slow for ARDT as Compared to MPS. 
MAURYA ET AL

Table 1. Simulation results for the variation of sample size $n$ and $\theta=0.2$

\begin{tabular}{|c|c|c|c|c|c|c|c|c|c|c|}
\hline$n$ & Qtl & MME & MLE & MPS & MLS & WLS & MOP & MCVM & ADT & ADRT \\
\hline \multirow[t]{4}{*}{5} & MSE & $0.00769^{5}$ & $0.007871^{6}$ & $0.00536^{1}$ & $0.009012^{8}$ & $0.008233^{7}$ & $0.005448^{2}$ & $0.009589^{9}$ & $0.006856^{4}$ & $0.005927^{3}$ \\
\hline & AED & $0.319291^{2}$ & $0.31924^{1}$ & $0.332499^{4}$ & $0.365936^{9}$ & $0.357915^{7}$ & $0.33808^{6}$ & $0.361976^{8}$ & $0.333651^{5}$ & $0.323^{3}$ \\
\hline & Dmax & $.088111^{2}$ & $0.088057^{1}$ & $0.091436^{4}$ & $0.100716^{9}$ & $0.09881^{7}$ & $0.092527^{6}$ & $0.099327^{8}$ & $0.092156^{5}$ & $0.089174^{3}$ \\
\hline & S Rank & $19^{4}$ & $18^{3}$ & $14^{1}$ & $41^{9}$ & $32^{7}$ & $23^{5}$ & $40^{8}$ & $24^{6}$ & $14^{1}$ \\
\hline \multirow{5}{*}{15} & BIAS & $0.008638^{7}$ & $0.009184^{8}$ & $-0.008128^{6}$ & $0.004232^{4}$ & $0.003596^{2}$ & $-0.010373^{9}$ & $0.006648^{5}$ & $0.003959^{3}$ & $0.001941^{1}$ \\
\hline & AED & $0.181445^{2}$ & $0.181013^{1}$ & $0.189446^{4}$ & $0.208996^{9}$ & $0.201689^{7}$ & $0.197231^{6}$ & $0.207922^{8}$ & $0.194808^{5}$ & $0.186107^{3}$ \\
\hline & Dabs & $0.072088^{2}$ & $0.071918^{1}$ & $0.075255^{4}$ & $0.08296^{9}$ & $0.080087^{7}$ & $0.078307^{6}$ & $0.08253^{8}$ & $0.077367^{5}$ & $0.073943^{3}$ \\
\hline & Dmax & $.049146^{2}$ & $0.049016^{1}$ & $0.051236^{4}$ & $0.056707^{9}$ & $0.054724^{7}$ & $0.053087^{6}$ & $0.056374^{8}$ & $0.052865^{5}$ & $0.050497^{3}$ \\
\hline & S Rank & $17^{3}$ & 162 & $19^{4}$ & $39^{9}$ & $30^{7}$ & $29^{6}$ & $38^{8}$ & $24^{5}$ & $13^{1}$ \\
\hline \multirow{3}{*}{30} & Dabs & $0.051036^{2}$ & $0.050905^{1}$ & $0.052817^{4}$ & $0.058498^{9}$ & $0.05595^{6}$ & $0.056007^{7}$ & $0.058362^{8}$ & $0.054893^{5}$ & $0.052356^{3}$ \\
\hline & Dmax & $034425^{2}$ & $0.034331^{1}$ & $0.035602^{4}$ & $0.039517^{9}$ & $0.037779^{7}$ & $0.037643^{6}$ & $0.03941^{8}$ & $0.037075^{5}$ & $0.035349^{3}$ \\
\hline & S Rank & $15^{3}$ & $12^{1}$ & $21^{4}$ & $39^{9}$ & $28^{6}$ & $34^{7}$ & $38^{8}$ & $24^{5}$ & $14^{2}$ \\
\hline \multirow[t]{6}{*}{60} & MSE & $0.000456^{3}$ & $0.000455^{2}$ & $0.000452^{1}$ & $0.00059^{8}$ & $0.000534^{7}$ & $0.000512^{5}$ & $0.000593^{9}$ & $0.000523^{6}$ & $0.000474^{4}$ \\
\hline & BIAS & $0.00217^{6}$ & $0.002299^{7}$ & $-0.003974^{8}$ & $0.001034^{4}$ & $0.001032^{3}$ & $-0.006037^{9}$ & $0.001659^{5}$ & $0.001014^{2}$ & $0.000517^{1}$ \\
\hline & AED & $0.089608^{2}$ & $0.089408^{1}$ & $0.091946^{3}$ & $0.10264^{9}$ & $0.097596^{6}$ & $0.098772^{7}$ & $0.102505^{8}$ & $0.096656^{5}$ & $0.092243^{4}$ \\
\hline & Dabs & $0.035944^{2}$ & $0.035864^{1}$ & $0.036881^{3}$ & $0.041159^{9}$ & $0.039141^{6}$ & $0.039612^{7}$ & $0.041105^{8}$ & $0.038765^{5}$ & $0.036999^{4}$ \\
\hline & Dmax & $0.024147^{2}$ & $0.024091^{1}$ & $0.024771^{3}$ & $0.027682^{9}$ & $0.026316^{6}$ & $0.026551^{7}$ & $0.02764^{8}$ & $0.026066^{5}$ & $0.024869^{4}$ \\
\hline & S Rank & $15^{2}$ & $12^{1}$ & $18^{4}$ & $39^{9}$ & $28^{6}$ & $35^{7}$ & $38^{8}$ & $23^{5}$ & $17^{3}$ \\
\hline
\end{tabular}




\section{COMPARATIVE STUDY OF NEW AND TRADITIONAL ESTIMATORS}

Table 2. Simulation results for the variation of sample size $n$ and $\theta=0.5$

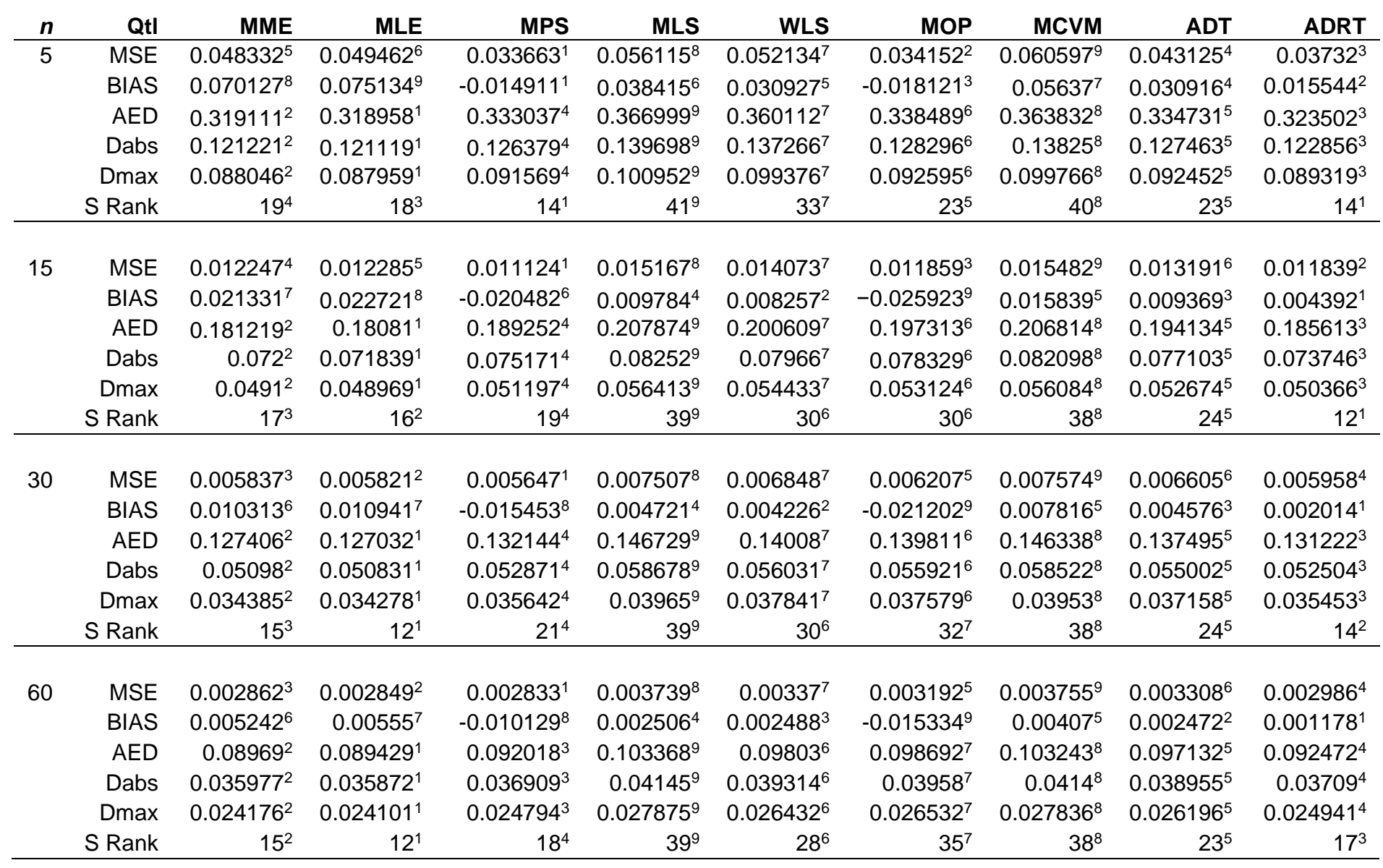


MAURYA ET AL

Table 3. Simulation results for the variation of sample size $n$ and $\theta=1$

\begin{tabular}{|c|c|c|c|c|c|c|c|c|c|c|}
\hline$n$ & Qtl & MME & MLE & MPS & MLS & WLS & MOP & MCVM & ADT & ADRT \\
\hline \multirow[t]{5}{*}{5} & MSE & $0.197017^{5}$ & $0.20137^{6}$ & $0.135909^{1}$ & $0.230542^{8}$ & $0.211519^{7}$ & $0.137782^{2}$ & $0.249869^{9}$ & $0.176621^{4}$ & $0.151213^{3}$ \\
\hline & BIAS & $0.144755^{8}$ & $0.154559^{9}$ & $-0.026393^{1}$ & $0.08299^{6}$ & $0.066019^{4}$ & $-0.03306^{2}$ & $0.119478^{7}$ & $0.067177^{5}$ & $0.034814^{3}$ \\
\hline & AED & $0.320737^{2}$ & $0.320538^{1}$ & $0.333397^{4}$ & $0.368385^{9}$ & $0.360106^{7}$ & $0.338693^{6}$ & $0.365693^{8}$ & $0.33614^{5}$ & $0.32451^{3}$ \\
\hline & Dmax & $0.088343^{2}$ & $0.088243^{1}$ & $0.091546^{4}$ & $0.101247^{9}$ & $0.099352^{7}$ & $0.09254^{5}$ & $0.10016^{8}$ & $0.092697^{6}$ & $0.089468^{3}$ \\
\hline & S Rank & $19^{4}$ & $18^{3}$ & $14^{1}$ & $41^{9}$ & $32^{7}$ & $21^{5}$ & $40^{8}$ & $25^{6}$ & $15^{2}$ \\
\hline \multirow{5}{*}{15} & BIAS & $0.043599^{7}$ & $0.046235^{8}$ & $-0.040358^{6}$ & $0.021701^{4}$ & $0.018283^{2}$ & $-0.051658^{9}$ & $0.033815^{5}$ & $0.020304^{3}$ & $0.009872^{1}$ \\
\hline & AED & $0.181222^{2}$ & $0.18089^{1}$ & $0.189296^{4}$ & $0.208179^{9}$ & $0.200994^{7}$ & $0.197519^{6}$ & $0.207137^{8}$ & $0.194154^{5}$ & $0.185783^{3}$ \\
\hline & Dabs & $0.071998^{2}$ & $0.071868^{1}$ & $0.075189^{4}$ & $0.082635^{9}$ & $0.079808^{7}$ & $0.078409^{6}$ & $0.082219^{8}$ & $0.077106^{5}$ & $0.073811^{3}$ \\
\hline & Dmax & $.049042^{2}$ & $0.048936^{1}$ & $51131^{4}$ & $0.056437^{9}$ & $0.054474^{7}$ & $0.053095^{6}$ & $0.056113^{8}$ & $0.052637^{5}$ & $0.050351^{3}$ \\
\hline & S Rank & $17^{3}$ & $16^{2}$ & $19^{4}$ & $39^{9}$ & $30^{7}$ & $29^{6}$ & $38^{8}$ & $24^{5}$ & $13^{1}$ \\
\hline \multirow{3}{*}{30} & Dabs & $0.051075^{2}$ & $0.050942^{1}$ & $2795^{4}$ & $0.058489^{9}$ & $0.055957^{6}$ & $0.055991^{7}$ & $0.058352^{8}$ & $0.054948^{5}$ & $0.052455^{3}$ \\
\hline & Dmax & $034459^{2}$ & $0.034363^{1}$ & $0.035607^{4}$ & $0.03952^{9}$ & $0.037794^{7}$ & $0.037634^{6}$ & $0.039413^{8}$ & $0.037116^{5}$ & $0.035427^{3}$ \\
\hline & S Rank & $15^{3}$ & $12^{1}$ & $21^{4}$ & $39^{9}$ & $28^{6}$ & $34^{7}$ & $38^{8}$ & $24^{5}$ & $14^{2}$ \\
\hline \multirow[t]{6}{*}{60} & MSE & $011541^{3}$ & $115^{2}$ & $434^{1}$ & 0.01 & $0.013485^{7}$ & $0.01296^{5}$ & $0.014947^{9}$ & $0.013224^{6}$ & $0.011974^{4}$ \\
\hline & BIAS & $0.010374^{6}$ & $0.011007^{7}$ & $-0.020334^{8}$ & $0.004572^{2}$ & $0.004674^{4}$ & $-0.030573^{9}$ & $0.007703^{5}$ & $0.004625^{3}$ & $0.002095^{1}$ \\
\hline & AED & $0.090191^{2}$ & $0.089984^{1}$ & $0.09254^{3}$ & $0.103246^{9}$ & $0.098182^{6}$ & $0.09944^{7}$ & $0.103109^{8}$ & $0.097235^{5}$ & $0.092702^{4}$ \\
\hline & Dabs & $0.036178^{2}$ & $0.036095^{1}$ & $0.037118^{3}$ & $0.041402^{9}$ & $0.039376^{6}$ & $0.039879^{7}$ & $0.041346^{8}$ & $0.038997^{5}$ & $0.037183^{4}$ \\
\hline & Dmax & $0.024309^{2}$ & $0.024253^{1}$ & $0.024935^{3}$ & $0.027842^{9}$ & $0.02647^{6}$ & $0.02674^{7}$ & $0.027801^{8}$ & $0.026221^{5}$ & $0.025001^{4}$ \\
\hline & S Rank & $15^{2}$ & $12^{1}$ & $18^{4}$ & $37^{8}$ & $29^{6}$ & $35^{7}$ & $38^{9}$ & $24^{5}$ & $17^{3}$ \\
\hline
\end{tabular}




\section{COMPARATIVE STUDY OF NEW AND TRADITIONAL ESTIMATORS}

Table 4. Simulation results for the variation of sample size $n$ and $\theta=2$

\begin{tabular}{|c|c|c|c|c|c|c|c|c|c|c|}
\hline$n$ & QtI & MME & MLE & MPS & MLS & WLS & MOP & MCVM & ADT & ADRT \\
\hline \multirow[t]{6}{*}{5} & MSE & 0.7766895 & 0.7944696 & 0.5387661 & 0.9088578 & 0.8191237 & 0.5465932 & 0.9804799 & 0.6960554 & 0.5989223 \\
\hline & BIAS & 0.2833378 & 0.3034419 & -0.0572581 & 0.1576616 & 0.1206114 & -0.0698353 & 0.2291097 & 0.1272885 & 0.0646920 \\
\hline & AED & 0.3190882 & 0.3188111 & 0.3323034 & 0.3667829 & 0.3574067 & 0.3376186 & 0.3637648 & 0.3350325 & 0.3232243 \\
\hline & Dabs & 0.1212172 & 0.1210761 & 0.1261254 & 0.1396679 & 0.1364297 & 0.1279866 & 0.1382668 & 0.1275875 & 0.1227783 \\
\hline & Dmax & 0.0880092 & 0.0878981 & 0.0913914 & 0.1009239 & 0.0987447 & 0.0923875 & 0.0997528 & 0.0924836 & 0.0892543 \\
\hline & S Rank & 194 & 183 & 141 & 419 & 327 & 225 & 408 & 256 & 141 \\
\hline \multirow[t]{6}{*}{15} & MSE & 0.1969064 & 0.1974775 & 0.1779871 & 0.2450478 & 0.2269947 & 0.1894692 & 0.2502939 & 0.2120956 & 0.1903013 \\
\hline & BIAS & 0.0880927 & 0.0934698 & -0.0797036 & 0.0437914 & 0.0372842 & -0.1021789 & 0.0680025 & 0.0410343 & 0.0206111 \\
\hline & AED & 0.1813572 & 0.1809491 & 0.1891634 & 0.2080879 & 0.2008657 & 0.1972056 & 0.2070538 & 0.1940165 & 0.1857873 \\
\hline & Dabs & 0.0720542 & 0.0718911 & 0.0751440 & 0.0825989 & 0.0797597 & 0.0782916 & 0.0821858 & 0.0770535 & 0.0738123 \\
\hline & Dmax & 0.0491082 & 0.0489741 & 0.0511444 & 0.0564249 & 0.0544587 & 0.0530516 & 0.0561058 & 0.0526235 & 0.0503813 \\
\hline & S Rank & 173 & 162 & 194 & 399 & 307 & 296 & 388 & 245 & 131 \\
\hline \multirow[t]{6}{*}{30} & MSE & 3233 & 852 & 0.0900831 & 868 & 9617 & 0.0991815 & 0.1202049 & 1286 & 0.0947434 \\
\hline & BIAS & 0.0426646 & 0.0452657 & -0.0603998 & 0.0205534 & 0.0186892 & -0.0836169 & 0.0329250 & 0.0198293 & 0.0097681 \\
\hline & AED & 0.1273682 & 0.1270321 & 0.1318264 & 0.1459729 & 0.1395826 & 0.1397767 & 0.1456348 & 0.1370250 & 0.1306243 \\
\hline & Dabs & 0.0509652 & 0.0508311 & 0.0527434 & 0.0583749 & 0.0558316 & 0.0559077 & 0.0582398 & 0.0548115 & 0.0522643 \\
\hline & Dmax & 0.0343822 & 0.0342851 & 0.0355740 & 0.0394439 & 0.0377127 & 0.0375826 & 0.0393388 & 0.0370275 & 0.0353043 \\
\hline & S Rank & 153 & 121 & 214 & 399 & 286 & 347 & 388 & 245 & 142 \\
\hline \multirow[t]{6}{*}{60} & MSE & 0.0459023 & 0.0456992 & 0.0454751 & 0.0595428 & 0.0538677 & 0.0513425 & 0.0597890 & 0.0528576 & 0.0478144 \\
\hline & BIAS & 0.0204566 & 0.0217670 & -0.0409378 & 0.0089752 & 0.0090574 & -0.0615739 & 0.0152350 & 0.0090193 & 0.0040761 \\
\hline & AED & 0.0898432 & 0.0895861 & 0.0923233 & 0.1031890 & 0.0980926 & 0.0989287 & 0.1030448 & 0.0971435 & 0.0925340 \\
\hline & Dabs & 0.0360382 & 0.0359351 & 0.0370323 & 0.0413759 & 0.0393460 & 0.0396747 & 0.0413280 & 0.0389650 & 0.0371144 \\
\hline & Dmax & 0.0242182 & 0.0241471 & 0.0248830 & 0.0278319 & 0.0264536 & 0.0266700 & 0.0277898 & 0.0262025 & 0.0249564 \\
\hline & S Rank & 152 & 121 & 184 & 378 & 296 & 357 & 389 & 245 & 173 \\
\hline
\end{tabular}




\section{MAURYA ET AL}

Table 5. Performance rank for the estimation procedures

\begin{tabular}{|c|c|c|c|c|c|c|c|c|c|c|}
\hline$n$ & $\theta$ & MME & MLE & MPS & MLS & WLS & MOP & MCVM & ADT & ADRT \\
\hline \multirow[t]{5}{*}{5} & 0.2 & 4 & 3 & 1 & 9 & 7 & 5 & 8 & 6 & 1 \\
\hline & 0.5 & 4 & 3 & 1 & 9 & 7 & 5 & 8 & 5 & 1 \\
\hline & 1.0 & 4 & 3 & 1 & 9 & 7 & 4 & 8 & 6 & 2 \\
\hline & 2.0 & 4 & 3 & 1 & 9 & 7 & 5 & 8 & 6 & 1 \\
\hline & Rank & $16^{4}$ & $12^{3}$ & $4^{1}$ & $36^{9}$ & $28^{7}$ & $19^{5}$ & $32^{8}$ & $23^{6}$ & $5^{2}$ \\
\hline \multirow[t]{5}{*}{15} & 0.2 & 3 & 2 & 4 & 9 & 7 & 6 & 8 & 5 & 1 \\
\hline & 0.5 & 3 & 2 & 4 & 9 & 6 & 6 & 8 & 5 & 1 \\
\hline & 1.0 & 3 & 2 & 4 & 9 & 7 & 6 & 8 & 5 & 1 \\
\hline & 2.0 & 3 & 2 & 4 & 9 & 7 & 6 & 8 & 5 & 1 \\
\hline & Rank & $12^{3}$ & $8^{2}$ & $16^{4}$ & $36^{9}$ & $27^{7}$ & $24^{6}$ & $32^{8}$ & $20^{5}$ & $4^{1}$ \\
\hline \multirow[t]{5}{*}{30} & 0.2 & 3 & 1 & 4 & 9 & 6 & 7 & 8 & 5 & 2 \\
\hline & 0.5 & 3 & 1 & 4 & 9 & 6 & 7 & 8 & 5 & 2 \\
\hline & 1.0 & 3 & 1 & 4 & 9 & 6 & 7 & 8 & 5 & 2 \\
\hline & 2.0 & 3 & 1 & 4 & 9 & 6 & 7 & 8 & 5 & 2 \\
\hline & Rank & $12^{3}$ & $4^{1}$ & $16^{4}$ & $36^{9}$ & $24^{6}$ & $28^{7}$ & $32^{8}$ & $20^{5}$ & $8^{2}$ \\
\hline \multirow[t]{5}{*}{60} & 0.2 & 2 & 1 & 4 & 9 & 6 & 7 & 8 & 5 & 3 \\
\hline & 0.5 & 2 & 1 & 4 & 9 & 6 & 7 & 8 & 5 & 3 \\
\hline & 1.0 & 2 & 1 & 4 & 8 & 6 & 7 & 9 & 5 & 3 \\
\hline & 2.0 & 2 & 1 & 4 & 8 & 6 & 7 & 9 & 5 & 3 \\
\hline & Rank & $8^{2}$ & $4^{1}$ & $16^{4}$ & $34^{8}$ & $24^{6}$ & $28^{7}$ & $34^{8}$ & $20^{5}$ & $12^{3}$ \\
\hline
\end{tabular}

Table 6. Overall performance of estimators

\begin{tabular}{rrrrrrrrrr}
$\boldsymbol{n}$ & MME & MLE & MPS & MLS & WLS & MOP & MCVM & ADT & ADRT \\
\hline 5 & 4 & 3 & 1 & 9 & 7 & 5 & 8 & 6 & 2 \\
15 & 3 & 2 & 4 & 9 & 7 & 6 & 8 & 5 & 1 \\
30 & 3 & 1 & 4 & 9 & 6 & 7 & 8 & 5 & 2 \\
60 & 2 & 1 & 4 & 8 & 6 & 7 & 8 & 5 & 3 \\
\hline
\end{tabular}

\section{Asymptotic Confidence Interval and Estimate of the Parameter}

In this section, we will discuss on asymptotic confidence interval, coverage probability, and average length of parameter and average estimate of the parameter. For large sample, estimated asymptotic confidence interval can be defined as

$$
\hat{\theta} \pm Z_{\eta / 2} \sqrt{\operatorname{var}(\hat{\theta})}
$$




\section{COMPARATIVE STUDY OF NEW AND TRADITIONAL ESTIMATORS}

where $Z_{\eta / 2}$ denotes the upper $\eta / 2 \%$ point of standard normal distribution. It may be interesting to see that if we estimate the var( ) through the simulated data and obtain the interval as stated above, whether it meets the desired confidence level. For this purpose, we have provided the average estimate of the parameter along with average confidence interval, its average length and coverage probability in Table 7 and 8 based on the considered nine estimation methods. In these tables, CI stands for asymptotic confidence interval, L stands for length of asymptotic confidence interval, and CP stands for coverage probability. We have also ranked the average length of confidence interval and these are presented in Table 9. Similarly, following a similar procedure as adopted in ranking the estimation method, the various methods have been ranked on basis of the average length of the confidence interval and the results are summarized for varying $n$ and $\theta$ in Table 10 .

- Tables 7-8 show that coverage probabilities are close to the prefixed $95 \%$ confidence level. It may also be noted that average length of confidence interval decreases as sample size increases.

- From Table 9, we observe that, the average length of confidence interval based on MOP is least for small sample size $(n=5)$ and for other sample sizes $(n=15,30,60)$ MPS is least, and it is noted to be longest for the interval based on MCVM except for $n=5$ and $\theta=0.2,0.5,1$. In these cases, the interval based on WLS is found to have the longest average length of the confidence interval. Also, the decreasing the length of CI results in decreases the $\mathrm{CP}$ for at least $95 \%$ level of confidence.

- It may also be noted from Table 9, MOP has second shortest length after MPS and MLE placed third position in ranking for all $\theta$ and $n=15,30$, and 60. It is important to mention here that the length of CI of MLE is second shortest length after MPS for large sample $(n=60)$.

- From Table 10, we see that the intervals based on MPS are found to have rank 1 and intervals based on MCVM have rank 9 for all the considered sample sizes. MOP has second shortest length of $\mathrm{CI}$ in all the considered methods. 


\section{MAURYA ET AL}

Table 7. Asymptotic confidence interval, coverage probability, length of interval and average estimate of parameter for $\theta=0.2$ and $\theta=0.5$

\begin{tabular}{|c|c|c|c|c|c|c|c|c|c|}
\hline$n \theta=0.2$ & MME & MLE & MPS & MLS & WLS & MOP & MCVM & ADT & ADRT \\
\hline $\mathrm{Cl}$ & $(0.0,0.49284)$ & לאר & $1888)$ & 3644 & $20212861)$ & 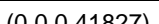 & 3579) & 3041 & 1608) \\
\hline
\end{tabular}
$\begin{array}{llllllllll}\mathrm{L}(\mathrm{CP}) & 0.49284(0.95829) & 0.4963(0.95815) & 0.41888(0.95811) & 0.63644(0.97149) & 2.12861(0.99855) & 0.41827(0.95804) & 0.63579(0.97044) & 0.47304(0.95905) & 0.44608(0.95828)\end{array}$

$15 \mathrm{Cl}(0.10626,0.3177)(0.10686,0.31817)(0.09788,0.29197)(0.08394,0.33424)(0.08781,0.3286)(0.09411,0.29129)(0.08554,0.33744)(0.09602,0.31935)(0.10056,0.31006)$ $\begin{array}{llllllllll}\text { L(CP) } & 0.21143(0.95535) & 0.2113(0.95547) & 0.19409(0.95499) & 0.2503(0.95648) & 0.24079(0.95691) & 0.19718(0.95503) & 0.2519(0.95627) & 0.22333(0.95487) & 0.2095(0.95509)\end{array}$

$30 \mathrm{Cl}(0.13549,0.27639)(0.13585,0.27653)(0.12875,0.26231) \quad(0.123,0.28574) \quad(0.1264,0.28165)(0.12454,0.26188)(0.12387,0.28735)(0.12868,0.27911)(0.13151,0.27391)$ $\begin{array}{llllllllll}L(C P) & 0.1409(0.95412) & 0.14068(0.95412) & 0.13356(0.95405) & 0.16274(0.95504) & 0.15525(0.95547) & 0.13734(0.95414) & 0.16349(0.95493) & 0.15044(0.95465) & 0.1424(0.95419)\end{array}$ $60 \mathrm{Cl}(0.15509,0.25075) \quad(0.1553,0.25079)(0.15045,0.24305) \quad(0.147,0.2572) \quad(0.14972,0.2543)(0.14643,0.24297)(0.14749,0.25797)(0.15044,0.25335)(0.15246,0.25018)$ \begin{tabular}{llllllllll}
$\mathrm{L}(\mathrm{CP})$ & $0.09567(0.95179)$ & $0.09548(0.95179)$ & $0.0926(0.95187)$ & $0.1102(0.95345)$ & $0.10459(0.95284)$ & $0.09654(0.95202)$ & $0.11048(0.95347)$ & $0.10291(0.9527)$ & $0.09772(0.95248)$ \\
\hline
\end{tabular}

\begin{tabular}{|c|c|c|c|c|c|c|c|c|c|c|}
\hline & $\theta=0.5$ & MME & MLE & MPS & MLS & WLS & MOP & MCVM & ADT & ADRT \\
\hline \multirow{2}{*}{5} & $\mathrm{Cl}$ & $(0.0,1.22411)$ & $(0.0,1.23321)$ & $(0.0,1.04063)$ & $(0.0,1.44465)$ & $(0.0,2.05718)$ & $(0.0,1.0395)$ & $(0.0,1.48829)$ & $(0.0,1.17287)$ & $(0.0,1.10971)$ \\
\hline & $\mathrm{L}(\mathrm{CP})$ & 1.22411(0.95766) & $1.23321(0.9579)$ & $1.04063(0.95746)$ & $1.44465(0.96443)$ & $2.05718(0.98609)$ & ) $1.0395(0.95747)$ & 1.48829(0.96447) & $1.17287(0.95799)$ & $1.10971(0.95788)$ \\
\hline \multirow{2}{*}{15} & $\mathrm{Cl}$ & I $(0.26578,0.79363)$ & $(0.26711,0.79504)$ & $(0.24456,0.72981)$ & $(0.21207,0.83131)$ & $(0.22207,0.81692)$ & ) $(0.23479,0.72884)$ & $(0.21611,0.83931)$ & $(0.24134,0.79575)$ & $(0.2518,0.77372)$ \\
\hline & $\mathrm{L}(\mathrm{CP})$ & $0.52785(0.95531)$ & $0.52793(0.95528)$ & $0.48524(0.95527)$ & $0.61924(0.95565)$ & $0.59486(0.95588)$ & $0.49405(0.95493)$ & $0.6232(0.95566)$ & $0.55441(0.95578)$ & $0.52192(0.95536)$ \\
\hline \multirow{2}{*}{30} & $\mathrm{Cl}$ & I $(0.33914,0.68937)$ & $(0.34009,0.68966)$ & $(0.32236,0.65419)$ & $(0.30732,0.71307)$ & $(0.316,0.7026)$ & $(0.31215,0.65294)$ & $(0.30949,0.7171)$ & $(0.32175,0.69629)$ & $(0.32903,0.68311)$ \\
\hline & $\mathrm{L}(\mathrm{CP})$ & $0.35023(0.95485)$ & $0.34957(0.95455)$ & $0.33183(0.9541)$ & $0.40575(0.9552)$ & $0.3866(0.95535)$ & ) $0.34079(0.95366)$ & $0.40761(0.95529)$ & $0.37454(0.95522)$ & $0.35408(0.95429)$ \\
\hline \multirow{2}{*}{60} & $\mathrm{Cl}$ & I $(0.38708,0.62723)$ & $(0.38767,0.62724)$ & $(0.37558,0.60785)$ & $(0.3666,0.64367)$ & $(0.37348,0.63636)$ & ) $(0.36577,0.60729)$ & $(0.36782,0.64559)$ & $(0.37523,0.6341)$ & $(0.38043,0.62597)$ \\
\hline & $\mathrm{L}(\mathrm{CP})$ & $0.24015(0.95162)$ & $0.23957(0.9519)$ & $0.23228(0.95184)$ & $0.27708(0.9531)$ & $0.26288(0.95288)$ & $0.24151(0.95207)$ & $0.27777(0.95307)$ & $0.25886(0.9527)$ & $0.24554(0.95226)$ \\
\hline
\end{tabular}




\section{COMPARATIVE STUDY OF NEW AND TRADITIONAL ESTIMATORS}

Table 8. Asymptotic confidence interval, coverage probability, length of interval and average estimate of parameter for $\theta=1$ and $\theta=2$

\begin{tabular}{rrrrrrrrrrr}
$n$ & $\boldsymbol{\theta = 1}$ & MME & MLE & MPS & MLS & WLS & MOP & MCVM & ADT & ADRT \\
\hline 5 & $\mathrm{Cl}$ & $(0.0,0.49284)$ & $(0.0,0.4963)$ & $(0.0,0.41888)$ & $(0.0,0.63644)$ & $(0.0,2.12861)$ & $(0.0,0.41827)$ & $(0.0,0.63579)$ & $(0.0,0.47304)$ & $(0.0,0.44608)$
\end{tabular}
$\begin{array}{llllllllll}\mathrm{L}(\mathrm{CP}) & 0.49284(0.95829) & 0.4963(0.95815) & 0.41888(0.95811) & 0.63644(0.97149) & 2.12861(0.99855) & 0.41827(0.95804) & 0.63579(0.97044) & 0.47304(0.95905) & 0.44608(0.95828)\end{array}$

$15 \mathrm{Cl}(0.10626,0.3177)(0.10686,0.31817)(0.09788,0.29197)(0.08394,0.33424)(0.08781,0.3286)(0.09411,0.29129)(0.08554,0.33744)(0.09602,0.31935)(0.10056,0.31006)$ $\begin{array}{llllllllll}\text { L(CP) } & 0.21143(0.95535) & 0.2113(0.95547) & 0.19409(0.95499) & 0.2503(0.95648) & 0.24079(0.95691) & 0.19718(0.95503) & 0.2519(0.95627) & 0.22333(0.95487) & 0.2095(0.95509)\end{array}$

$30 \mathrm{Cl}(0.13549,0.27639)(0.13585,0.27653)(0.12875,0.26231) \quad(0.123,0.28574) \quad(0.1264,0.28165)(0.12454,0.26188)(0.12387,0.28735)(0.12868,0.27911)(0.13151,0.27391)$ $\begin{array}{llllllllll}\mathrm{L}(\mathrm{CP}) & 0.1409(0.95412) & 0.14068(0.95412) & 0.13356(0.95405) & 0.16274(0.95504) & 0.15525(0.95547) & 0.13734(0.95414) & 0.16349(0.95493) & 0.15044(0.95465) & 0.1424(0.95419)\end{array}$ $60 \mathrm{Cl}(0.15509,0.25075) \quad(0.1553,0.25079)(0.15045,0.24305) \quad(0.147,0.2572) \quad(0.14972,0.2543)(0.14643,0.24297)(0.14749,0.25797)(0.15044,0.25335)(0.15246,0.25018)$ \begin{tabular}{llllllllll}
$\mathrm{L}(\mathrm{CP})$ & $0.09567(0.95179)$ & $0.09548(0.95179)$ & $0.0926(0.95187)$ & $0.1102(0.95345)$ & $0.10459(0.95284)$ & $0.09654(0.95202)$ & $0.11048(0.95347)$ & $0.10291(0.9527)$ & $0.09772(0.95248)$ \\
\hline
\end{tabular}

\begin{tabular}{|c|c|c|c|c|c|c|c|c|c|c|}
\hline$\underline{n}$ & $\theta=2$ & MME & MLE & MPS & MLS & WLS & MOP & MCVM & ADT & ADRT \\
\hline \multirow[t]{2}{*}{5} & $\mathrm{Cl}$ & $(0.0,1.22411)$ & $(0.0,1.23321)$ & $(0.0,1.04063)$ & $(0.0,1.44465)$ & $(0.0,2.05718)$ & $(0.0,1.0395)$ & $(0.0,1.48829)$ & $(0.0,1.17287)$ & $(0.0,1.10971)$ \\
\hline & L(CP) & $1.22411(0.95766)$ & $1.23321(0.9579)$ & $1.04063(0.95746)$ & $1.44465(0.96443)$ & $2.05718(0.98609)$ & $1.0395(0.95747)$ & $1.48829(0.96447)$ & $1.17287(0.95799)$ & $1.10971(0.95788)$ \\
\hline \multirow[t]{2}{*}{15} & $\mathrm{Cl}$ & $(0.26578,0.79363)$ & $(0.26711,0.79504)$ & $(0.24456,0.72981)$ & $(0.21207,0.83131)$ & $(0.22207,0.81692)$ & $(0.23479,0.72884)$ & $(0.21611,0.83931)$ & $(0.24134,0.79575)$ & $(0.2518,0.77372)$ \\
\hline & $\mathrm{L}(\mathrm{CP})$ & $0.52785(0.95531)$ & $0.52793(0.95528)$ & $0.48524(0.95527)$ & $0.61924(0.95565)$ & $0.59486(0.95588)$ & $0.49405(0.95493)$ & $0.6232(0.95566)$ & $0.55441(0.95578)$ & $0.52192(0.95536)$ \\
\hline \multirow[t]{2}{*}{30} & $\mathrm{Cl}$ & $(0.33914,0.68937)$ & $(0.34009,0.68966)$ & $(0.32236,0.65419)$ & $(0.30732,0.71307)$ & $(0.316,0.7026)$ & $(0.31215,0.65294)$ & $(0.30949,0.7171)$ & $(0.32175,0.69629)$ & $(0.32903,0.68311)$ \\
\hline & $\mathrm{L}(\mathrm{CP})$ & $0.35023(0.95485)$ & $0.34957(0.95455)$ & $0.33183(0.9541)$ & $0.40575(0.9552)$ & $0.3866(0.95535)$ & $0.34079(0.95366)$ & $0.40761(0.95529)$ & $0.37454(0.95522)$ & $0.35408(0.95429)$ \\
\hline \multirow[t]{2}{*}{60} & $\mathrm{Cl}$ & $(0.38708,0.62723)$ & $(0.38767,0.62724)$ & $(0.37558,0.60785)$ & $(0.3666,0.64367)$ & $(0.37348,0.63636)$ & $(0.36577,0.60729)$ & $(0.36782,0.64559)$ & $(0.37523,0.6341)$ & $(0.38043,0.62597)$ \\
\hline & $\mathrm{L}(\mathrm{CP})$ & $0.24015(0.95162)$ & $0.23957(0.9519)$ & $0.23228(0.95184)$ & $0.27708(0.9531)$ & $0.26288(0.95288)$ & $0.24151(0.95207)$ & $0.27777(0.95307)$ & $0.25886(0.9527)$ & $0.24554(0.95226)$ \\
\hline
\end{tabular}




\section{MAURYA ET AL}

Table 9. Performance rank for average confidence length

\begin{tabular}{|c|c|c|c|c|c|c|c|c|c|c|}
\hline$n$ & $\theta$ & MME & MLE & MPS & MLS & WLS & MOP & MCVM & ADT & ADRT \\
\hline \multirow[t]{5}{*}{5} & 0.2 & 5 & 6 & 2 & 8 & 9 & 1 & 7 & 4 & 3 \\
\hline & 0.5 & 5 & 6 & 2 & 7 & 9 & 1 & 8 & 4 & 3 \\
\hline & 1.0 & 5 & 6 & 2 & 7 & 9 & 1 & 8 & 4 & 3 \\
\hline & 2.0 & 5 & 6 & 2 & 8 & 7 & 1 & 9 & 4 & 3 \\
\hline & Rank & $20^{5}$ & $24^{6}$ & $8^{2}$ & $30^{7}$ & $34^{9}$ & $4^{1}$ & $32^{8}$ & $16^{4}$ & $12^{3}$ \\
\hline \multirow[t]{5}{*}{15} & 0.2 & 5 & 4 & 1 & 8 & 7 & 2 & 9 & 6 & 3 \\
\hline & 0.5 & 4 & 5 & 1 & 8 & 7 & 2 & 9 & 6 & 3 \\
\hline & 1.0 & 5 & 4 & 1 & 8 & 7 & 2 & 9 & 6 & 3 \\
\hline & 2.0 & 5 & 4 & 1 & 8 & 7 & 2 & 9 & 6 & 3 \\
\hline & Rank & $19^{5}$ & $17^{4}$ & $4^{1}$ & $32^{8}$ & $28^{7}$ & $8^{2}$ & $36^{9}$ & $24^{6}$ & $12^{3}$ \\
\hline \multirow[t]{5}{*}{30} & 0.2 & 4 & 3 & 1 & 8 & 7 & 2 & 9 & 6 & 5 \\
\hline & 0.5 & 4 & 3 & 1 & 8 & 7 & 2 & 9 & 6 & 5 \\
\hline & 1.0 & 4 & 3 & 1 & 8 & 7 & 2 & 9 & 6 & 5 \\
\hline & 2.0 & 4 & 3 & 1 & 8 & 7 & 2 & 9 & 6 & 5 \\
\hline & Rank & $16^{4}$ & $12^{3}$ & $4^{1}$ & $32^{8}$ & $28^{7}$ & $8^{2}$ & $36^{9}$ & $24^{6}$ & $20^{5}$ \\
\hline \multirow[t]{5}{*}{60} & 0.2 & 3 & 2 & 1 & 8 & 7 & 4 & 9 & 6 & 5 \\
\hline & 0.5 & 3 & 2 & 1 & 8 & 7 & 4 & 9 & 6 & 5 \\
\hline & 1.0 & 3 & 2 & 1 & 8 & 7 & 4 & 9 & 6 & 5 \\
\hline & 2.0 & 3 & 2 & 1 & 8 & 7 & 4 & 9 & 6 & 5 \\
\hline & Rank & $12^{3}$ & $8^{2}$ & $4^{1}$ & $32^{8}$ & $28^{7}$ & $16^{4}$ & $36^{9}$ & $24^{6}$ & $20^{5}$ \\
\hline
\end{tabular}

Table 10. Overall performance of average confidence length

\begin{tabular}{rrrrrrrrrr}
$\boldsymbol{N}$ & MME & MLE & MPS & MLS & WLS & MOP & MCVM & ADT & ADRT \\
\hline 5 & 5 & 6 & 2 & 7 & 9 & 1 & 8 & 4 & 3 \\
15 & 5 & 4 & 1 & 8 & 7 & 2 & 9 & 6 & 3 \\
30 & 4 & 3 & 1 & 8 & 7 & 2 & 9 & 6 & 5 \\
60 & 3 & 2 & 1 & 8 & 7 & 4 & 9 & 6 & 5 \\
O Rank & $17^{5}$ & $15^{3}$ & $5^{1}$ & $31^{8}$ & $30^{7}$ & $9^{2}$ & $35^{9}$ & $22^{6}$ & $16^{4}$ \\
\hline
\end{tabular}

\section{Conclusion}

In the present article, we have considered nine different classical estimation techniques for estimating the unknown lifetime characteristic from the Logarithmic Transformed Exponential distribution. Here, the considered estimation methods are, namely, Method of moments, method of maximum likelihood estimate, maximum product spacing method, least squares estimation, weighted least squares estimation, method of percentile, method of Cramer von-Mises, Anderson-Darling and Right- 


\section{COMPARATIVE STUDY OF NEW AND TRADITIONAL ESTIMATORS}

tailed Anderson-Darling. The performance of the estimators has been compared on the basis of bias, mean squared error, absolute difference between true and estimated cdf, maximum absolute difference between true and estimated distribution function, and average entropy difference. Asymptotic confidence intervals have been simulated on the basis of the estimates obtained through the above-mentioned methods and coverage probabilities and average confidence intervals have been obtained. The methods are then ranked from 1 to 9 for best to worst.

On the basis of the rankings, we may conclude that MPS method is the best for small sample size $(n=5)$, although for this situation ARDT also performs more are less equally well. However, for the interval estimation, for $n=15$, the ARDT is found to be the best. For other sample sizes, the MLE performs the best. But MPS has shortest average length confidence interval for all the considered situations. The intervals based on MLE are ranked at 3. From the above discussions, we may conclude that for the estimation of the parameter of the Logarithmic Transformed Exponential distribution, we may use MPS or ADRT method for small sample (sample size less than 15). For samples of larger sizes (i.e., $n>15$ ), MLE method may be recommended for use. However, if one is interested in the interval estimation, asymptotic confidence interval may be obtained by the use of MPS method.

\section{References}

Anderson, T. W. (1962). On the distribution of the two-sample Cramér-von Mises criterion. The Annals of Mathematical Statistics, 33(3), 1148-1159. doi: 10.1214/aoms/1177704477

Bakouch, H. S., Dey, S., Ramos, P. L., \& Louzada, F. (2017). Binomialexponential 2 distribution: Different estimation methods with weather applications. TEMA (São Carlos), 18(2), 233-251. doi:

10.5540/tema.2017.018.02.0233

Balakrishnan, N. (2014). Continuous multivariate distributions. In Wiley StatsRef: Statistics Reference Online. John Wiley \& Sons, Inc. doi: 10.1002/9781118445112.stat01249

Barlow, R. E., \& Proschan, F. (1975). Statistical theory of reliability and life testing: Probability models (Accession no. ADA006399). Fort Belvoir, VA: Defense Technical Information Center. 


\section{MAURYA ET AL}

Basu, S., Singh, S. K., \& Singh, U. (2018). Bayesian inference using product of spacings function for progressive hybrid type-I censoring scheme. Statistics, 52(2), 345-363. doi: 10.1080/02331888.2017.1405419

Boos, D. D. (1981). Minimum distance estimators for location and goodness of fit. Journal of the American Statistical Association, 76(375), 663-670. doi: 10.1080/01621459.1981.10477701

Boos, D. D. (1982). Minimum Anderson-Darling estimation. Communications in Statistics - Theory and Methods, 11(24), 2747-2774. doi: 10.1080/03610928208828420

Casella, C., \& Berger, R. L. (2002). Statistical inference (Vol. 2). Pacific Grove, CA: Duxbury.

Cheng, R. C. H., \& Amin, N. A. K. (1983). Estimating parameters in continuous univariate distributions with a shifted origin. Journal of the Royal Statistical Society: Series B (Methodological), 45(3), 394-403. doi: 10.1111/j.2517-6161.1983.tb01268.x

Choi, K., \& Bulgren, W. G. (1968). An estimation procedure for mixtures of distributions. Journal of the Royal Statistical Society: Series B (Methodological), 30(3), 444-460. doi: 10.1111/j.2517-6161.1968.tb00743.x

Coolen, F. P. A., \& Newby, M. J. (1990). A note on the use of the product of spacings in Bayesian inference. Kwantitatieve Methoden, 37, 19-32.

Gupta, R. D., \& Kundu, D. (1999). Theory \& methods: Generalized exponential distributions. Australian \& New Zealand Journal of Statistics, 41(2), 173-188. doi: 10.1111/1467-842X.00072

Gupta, R. D., \& Kundu, D. (2001a). Exponentiated exponential family: An alternative to gamma and Weibull distributions. Biometrical Journal, 43(1), $117-$ 130. doi: 10.1002/1521-4036(200102)43:1<117::AID-BIMJ117>3.0.CO;2-R

Gupta, R. D., \& Kundu, D. (2001b). Generalized exponential distribution: Different method of estimations. Journal of Statistical Computation and Simulation, 69(4), 315-337. doi: 10.1080/00949650108812098

Gupta, R. D., \& Kundu, D. (2007). Generalized exponential distribution: Existing results and some recent developments. Journal of Statistical Planning and Inference, 137(11), 3537-3547. doi: 10.1016/j.jspi.2007.03.030

Jaynes, E. T. (1968). Prior probabilities. IEEE Transactions on Systems Science and Cybernetics, 4(3), 227-241. doi: 10.1109/TSSC.1968.300117 


\section{COMPARATIVE STUDY OF NEW AND TRADITIONAL ESTIMATORS}

Kao, J. H. K. (1958). Computer methods for estimating Weibull parameters in reliability studies. IRE Transactions on Reliability and Quality Control, PGRQC-13, 15-22. doi: 10.1109/IRE-PGRQC.1958.5007164

Kao, J. H. K. (1959). A graphical estimation of mixed Weibull parameters in life-testing of electron tubes. Technometrics, 1(4), 389-407. doi: 10.1080/00401706.1959.10489870

Kaushik, A., Singh, U., \& Singh, S. K. (2017). Bayesian inference for the parameters of Weibull distribution under progressive type-I interval censored data with beta-binomial removals. Communications in Statistics - Simulation and Computation, 46(4), 3140-3158. doi: 10.1080/03610918.2015.1076469

Kumar, D., Singh, U., \& Singh, S. K. (2015). A method of proposing new distribution and its application to bladder cancer patient data. Journal of Statistics Applications \& Probability Letters, 2(3), 235-245. Retrieved from http://www.naturalspublishing.com/Article.asp?ArtcID=9686

Kumaraswamy, P. (1980). A generalized probability density-function for double-random processes. Journal of Hydrology, 46(1-2), 79-88. doi: 10.1016/0022-1694(80)90036-0

Laio, F. (2004). Cramer-von Mises and Anderson-Darling goodness of fit tests for extreme value distributions with unknown parameters. Water Resources Research, 40(9), 1-10. doi: 10.1029/2004WR003204

Larson, H. J. (1969). Introduction to probability theory and statistical inference. New York: Wiley.

Levine, R. D., \& Tribus, M. (1979). Maximum entropy formalism. Cambridge, MA: MIT Press.

Liu, J. (2007). Information theoretic content and probability [Unpublished doctoral thesis]. University of Florida.

Louzada, F., Ramos, P. L., \& Perdona, G. S. C. (2016). Different estimation procedures for the parameters of the extended exponential geometric distribution for medical data. Computational and Mathematical Methods in Medicine, 2016, 8727951. doi: 10.1155/2016/8727951

Luceno, A. (2006). Fitting the generalized Pareto distribution to data using maximum goodness-of-fit estimators. Computational Statistics \& Data Analysis, 51(2), 904-917. doi: 10.1016/j.csda.2005.09.011

Macdonald, P. D. M. (1971). Comment on "An estimation procedure for mixtures of distributions" by Choi and Bulgren. Journal of the Royal Statistical 


\section{MAURYA ET AL}

Society: Series B (Methodological), 33(2), 326-329. doi: 10.1111/j.2517-

6161.1971.tb00884.x

Mann, N. R., Singpurwalla, N. D., \& Schafer, R. E. (1974). Methods for statistical analysis of reliability and life data. New York, Wiley.

Maurya, S. K., Kaushik, A., Singh, R. K., Singh, S. K., \& Singh, U. (2016). A new method of proposing distribution and its application to real data. Imperial Journal of Interdisciplinary Research, 2(6), 1331-1338.

Maurya, S. K., Kaushik, A., Singh, S. K., \& Singh, U. (2017). A new class of distribution having decreasing, increasing, and bathtub-shaped failure rate. Communications in Statistics - Theory and Methods, 46(20), 10359-10372. doi: 10.1080/03610926.2016.1235196

Mazucheli, J., Ghitany, M. E., \& Louzada, F. (2017). Comparisons of ten estimation methods for the parameters of Marshall-Olkin extended exponential distribution. Communications in Statistics - Simulation and Computation, 46(7), 5627-5645. doi: 10.1080/03610918.2016.1171345

Mudholkar, G. S., Srivastava, D. K., \& Kollia, G. D. (1993). Exponentiated Weibull family for analyzing bathtub failure-rate data. IEEE Transactions on Reliability, 42(2), 299-302. doi: 10.1109/24.229504

Quandt, R. E., \& Ramsey, J. B. (1978). Estimating mixtures of normal distributions and switching regressions. Journal of the American statistical Association, 73(364), 730-738. doi: 10.1080/01621459.1978.10480085

R Core Team. (2018). R: A language and environment for statistical computing. Vienna, Austria: R Foundation for Statistical Computing. Retrieved from https://www.r-project.org/

Rahman, M., \& Pearson, L. M. (2001). Estimation in two-parameter exponential distributions. Journal of Statistical Computation and Simulation, 70(4), 371-386. doi: 10.1080/00949650108812128

Ranneby, B. (1984). The maximum spacing method. an estimation method related to the maximum likelihood method. Scandinavian Journal of Statistics, 11(2), 93-112.

Shannon, C. E. (1951). Prediction and entropy of printed English. Bell System Technical Journal, 30(1), 50-64. doi: 10.1002/j.15387305.1951.tb01366.x

Sharma, V. K., Singh, S. K., Singh, U., \& Ul-Farhat, K. (2017). Bayesian estimation on interval censored Lindley distribution using Lindley's 


\section{COMPARATIVE STUDY OF NEW AND TRADITIONAL ESTIMATORS}

approximation. International Journal of System Assurance Engineering and Management, 8(2), 799-810. doi: 10.1007/s13198-016-0528-x

Singh, R. K., Singh, S. K., \& Singh, U. (2016). Maximum product spacings method for the estimation of parameters of generalized inverted exponential distribution under progressive type II censoring. Journal of Statistics and Management Systems, 19(2), 219-245. doi: 10.1080/09720510.2015.1023553

Singh, R. K., Yadav, A. S., Singh, S. K., \& Singh, U. (2016). MarshallOlkin extended exponential distribution: Different method of estimations. Journal of Advanced Computing, 5(1), 12-28.

Singh, U., Singh, S. K., \& Singh, R. K. (2014a). Product spacings as an alternative to likelihood for Bayesian inferences. Journal of Statistics Applications \& Probability, 3(2), 179-188. Retrieved from http://www.naturalspublishing.com/Article.asp?ArtcID=6325

Singh, U., Singh, S. K., \& Singh, R. K. (2014b). A comparative study of traditional estimation methods and maximum product spacings method in generalized inverted exponential distribution. Journal of Statistics Applications \& Probability, 3(2), 153-169. Retrieved from http://www.naturalspublishing.com/Article.asp?ArtcID=6418

Singh, V. P., \& Rajagopal, A. K. (1986). A new method of parameter estimation for hydrologic frequency analysis. Hydrological Science and Technology, 2(3), 33-40.

Swain, J. J., Venkatraman, S., \& Wilson, J. R. (1988). Least-squares estimation of distribution functions in Johnson's translation system. Journal of Statistical Computation and Simulation, 29(4), 271-297. doi: 10.1080/00949658808811068

Tang, Q. (2014). Comparison of different methods for estimating log-normal means [Unpublished doctoral thesis]. East Tennessee State University.

Yari, G., \& Tondpour, Z. (2017). Estimation of the exponential Pareto II distribution parameters. Communications in Statistics - Simulation and Computation, 46(9), 6889-6906. doi: 10.1080/03610918.2016.1217012 